\title{
FASN Knockdown Inhibited Anoikis Resistance of Gastric Cancer Cells via P-ERK1/2/Bcl-xL Pathway
}

\author{
Li Yu $\mathbb{D}{ }^{1}$ Xin Wang $\mathbb{D},{ }^{1}$ Yao Du $\mathbb{D},{ }^{1}$ Xiaowen Zhang $\mathbb{D},{ }^{1}$ and Yunzhi Ling $\mathbb{D}^{2}$ \\ ${ }^{1}$ Center of Clinical Laboratory Diagnostics, Bengbu Medical College, Bengbu City, Anhui Province 233030, China \\ ${ }^{2}$ Department of Anesthesiology, The First Affiliated Hospital of Bengbu Medical College, Bengbu City, Anhui Province 233000, China
}

Correspondence should be addressed to Yunzhi Ling; yunzhil_lzhy@163.com

Received 8 December 2020; Accepted 3 July 2021; Published 19 August 2021

Academic Editor: Lukas J.A.C. Hawinkels

Copyright ( $2021 \mathrm{Li} \mathrm{Yu}$ et al. This is an open access article distributed under the Creative Commons Attribution License, which permits unrestricted use, distribution, and reproduction in any medium, provided the original work is properly cited.

\begin{abstract}
Anoikis resistance (AR) is a crucial step in tumor metastasis. The overexpression of fatty acid synthase (FASN) is not only related to the AR of osteosarcoma cells, but also evidenced on gastric cancer (GC). This study investigated the role of FASN in the AR of GC cells. Plates coated with poly-HEMA were used for the culture of cells with AR. Small interfering RNA targeting FASN (siFASN) was transfected into MNK-45 and AGS cells. The number and apoptosis of cells were assessed by a hemacytometer and Annexin-V-FITC/PI assay, respectively. Aggregated cells and colony numbers were manually counted under a microscope. The migration and invasion rates were measured via wound healing and Transwell invasion assays, respectively. The levels of FASN, phosphorylated (p)-ERK1/2, ERK1/2 and Bcl-xL were detected through western blot or quantitative reverse transcriptionpolymerase chain reaction (qRT-PCR). The results showed that the cell numbers of $M N K-45$ and $A G S$ were increased while that of GES-1 cell was decreased during the culture in suspension. A higher apoptosis rate and a smaller number of aggregated cells were observed in GES-1 cells in comparison with MNK-45 and AGS cells. A larger colony number, greater migration and invasion rates, and higher mRNA and protein expressions of FASN were presented in the AR group compared with the control group. Cells transfected with siFASN possessed lower migration and invasion rates, reduced expressions of FASN mRNA and protein, p-ERK1/2 and Bcl-xL, and induced a significantly declined ratio of p-ERK1/2 to ERK1/2. These findings suggest that down-regulation of FASN suppresses the AR of GC cells, which may be related to the inhibition of p-ERK1/2/Bcl-xL pathway.
\end{abstract}

\section{Introduction}

Gastric cancer (GC) is one of the most common malignant tumors in the world and ranks the third leading cause of cancer-related mortality, following lung and liver cancer [1]. Despite the decreased prevalence owing to the progresses made in medical technology, the prognosis of GC still remains poor, with a 5 -year survival rate of less than $30 \%$ [2]. And metastasis is the main cause of deaths in patients with GC [3]. As a kind of programmed cell death which responds to the detachment of cells from the original extracellular matrix (ECM), anoikis can prevent cells from growth and reattachment in a different site, thus blocking the colonization of cells in distant organs. Unlike the normal cells, cancer cells show a resistance against anoikis, which contribute to the development and metastasis of tumors $[4,5]$. Therefore, studies on the anoikis resistance (AR) of GC cells are of considerable significance for the prevention and treatment of metastasis in GC and the reduction on GC-induced mortality.

Fatty acid synthase (FASN) is a main enzyme participating in the formation of the newborn fat and is overexpressed in tissues of various tumors, such as prostate cancer and bone tumor [6-9]. Previous studies have proved an evidence on the interaction between FASN and the process of epithelialmesenchymal transition (EMT) in GC [10], which reveals that FASN may be a promising therapeutic target for the anti-cancer therapy. However, studies on the detailed effect of FASN on GC cells with AR remain inadequate. Moreover, FASN was shown to mediate breast cancer through the regulation of ERK1/2 [11, 12]. Extracellular regulated protein kinases (ERK), including ERK1 and ERK2, can be activated into phosphorylated (p)-ERK so as to promote the multiplication and suppress the apoptosis in the cells. Previous 
studies also revealed the relation on the activation of ERK activation and the cancer and AR [13-15]. Bcl-xL, an antiapoptotic member of the B-cell lymphoma-2 (Bcl-2) family, exerts a protective effect on cancer cells, including gastric cancer cells, as reported in previous publications [16-18]. Besides, the upregulation of FASN was proved to be associated with the AR of osteosarcoma cells via $\mathrm{p}$-ERK1/2/Bcl$x L$ [19]. Consequently, we tried to explore whether FASN realizes its effect on AR via ERK1/2/Bcl-xL pathway in GC.

In this study, the anoikis resistant GC cells were cultured in vitro, the purpose of which was to explore the biological characteristics of AR. In addition, the role and molecular mechanism of FASN in GC cells with AR were also investigated in vitro by controlling variables, the results of which demonstrated that FASN mediated AR in GC cells through p-ERK1/2/Bcl-xL pathway, making FASN as a promising therapeutic target for the intervention and remedy of metastasis in GC.

\section{Materials and Methods}

2.1. Cell Culture. Normal gastric mucosa epithelial cell line GES-1 and GC cell lines MNK-45 and AGS were obtained from Shanghai Institutes for Biological Sciences (Shanghai, China) and were cultured in RPMI-1640 medium (E600028-0500, Sangon Biotech Co., Ltd., Shanghai, China) which was supplemented with $10 \%$ fetal bovine serum (FBS; E600001-0500, Sangon Biotech Co., Ltd., Shanghai, China), 1\% L-glutamine (A600224-0500, Sangon Biotech Co., Ltd., Shanghai, China) and Benzylpenicillin/streptomycin (B540732-0010, Sangon Biotech Co., Ltd., Shanghai, China) at $37^{\circ} \mathrm{C}$ in an incubator containing $5 \% \mathrm{CO}_{2}$.

2.2. Suspension Culture of Anoikis Resistant GC Cells. The adherent cells were trypsinized (A610681-0100, Sangon Biotech Co., Ltd., Shanghai, China) so as to obtain the single cell suspension and then seeded at $10^{6}$ per well in Costar ultralow attachment 6-well plates (3471, Corning Incorporation, Corning, NY, USA). Poly-hydroxyethyl methacrylate (polyHEMA, ST1582, Beyotime Biotechnology, Shanghai, China) was pre-dissolved in 95\% ethanol (493511, Sigma-Aldrich, Ontario, Canada), and the solution was added into the culture plates to induce the anoikis in the cell. The cells were passaged every three days. After continuous passages, the anoikis resistant cells were selected and collected as AR group for biological characteristics analysis. Cells under adherent culture were used as the control group and received no treatment.

2.3. Suspension Culture and Detection. The adherent cells of the control and AR groups were trypsinized to the single cell suspension and then seeded at $10^{6}$ per well in costar ultra-low attachment 24-well plates (3473, Corning Incorporation, Corning, NY, USA). A hemacytometer (AMQAF1000, Thermo Fisher Scientific, Waltham, MA, USA) was used to count the numbers of cells on days $0,2,4$ and 6 . The morphology of cells was observed using a phase contrast microscope (magnification: 100x; DYS-810, Shanghai Dianying Optical Instrument Co., Ltd., Shanghai, China).
TABLE 1: quantitative reverse transcription-polymerase chain reaction (qRT-PCR) primers.

\begin{tabular}{lcc}
\hline Target gene & Forward primers, 5'-3' & Reverse primers, 5'-3' \\
\hline \multirow{2}{*}{$\beta$-Actin } & CTCCATCCTGGCCTCG & GCTGTCACCTTCACCG \\
& CTGT & TTCC \\
\multirow{2}{*}{ FASN } & CAACTCACGCTCCG & TGTGGATGCTGTCA \\
& GAAA & AGGG \\
\hline
\end{tabular}

2.4. Cell Apoptosis Assay. After the culture in suspension for 24 hours (h), the apoptosis of adherent and detached cells was assessed by an Annexin-V-FITC Apoptosis Detection Kit with PI (E606336-0500, Sangon Biotech Co., Ltd., Shanghai, China). The cells were washed 3 times with PBS and then resuspended in binding buffer. Annexin V-FITC and PI were added to the solution containing cells in a dark room at room temperature for 15 minutes ( $\mathrm{min}$ ). The apoptosis rates were analyzed in a flow cytometer (CytoFLEX, Beckman Coulter, Brea, CA, USA).

2.5. Colony Formation Assay. Cells in logarithmic phase were trypsinized to single cell suspension and then seeded at $10^{5}$ per well in Costar ultra-low attachment 24-well plates for 7 days of suspension culture. Then the cell suspension was seeded into normal 24-well plates (J00240, Shanghai Jinan Biological Technology Co., Ltd., Shanghai, China) until the formation of colonies. After being fixed with $4 \%$ paraformaldehyde (E672002-0500, Sangon Biotech Co., Ltd., Shanghai, China) at room temperature for $15 \mathrm{~min}$ and stained with crystal violet (E607309-0100, Sangon Biotech Co., Ltd., Shanghai, China) for $15 \mathrm{~min}$, the colonies were manually counted.

2.6. Wound Healing Assay. Cells were seeded in 6-well plates (J00660, Shanghai Jinan Biological Technology Co., Ltd., Shanghai, China) at a density of $5 \times 10^{6}$ cells per well and cultured until $80-90 \%$ confluence. A pipette (Sangon Biotech Co., Ltd., Shanghai, China) tip was used to make a straight scratch which simulated as a wound. Cells were rinsed with PBS and the medium was replaced with serum-free medium at $37^{\circ} \mathrm{C}$ for $24 \mathrm{~h}$. Images were observed and recorded at 0 and $24 \mathrm{~h}$ by a DYS- 810 microscope (magnification: $100 \mathrm{x}$; Shanghai Dianying Optical Instrument Co., Ltd., Shanghai, China) and the distance for the migration was measured by Image J, version 1.48 (National Center for Biotechnology Information, Bethesda, MD, USA). Six visual fields were randomly selected from each room.

2.7. Transwell Invasion Assay. Cell invasion assay was performed in a 24-well Transwell chamber (3379, Corning Incorporation, Corning, NY, USA). The upper filters were pre-coated with Matrigel (354234, Corning Incorporation, Corning, NY, USA), and cells with serum-free medium were seeded into the upper chambers at a density of $4 \times 10^{5} \mathrm{cell} / \mathrm{ml}$. The medium in the lower chamber contained 10\% FBS and functioned as a source of chemoattractants. After the incubation at $37^{\circ} \mathrm{C}$ for $24 \mathrm{~h}$, cells that passed through the Matrigelcoated membrane were fixed with $4 \%$ paraformaldehyde at $4^{\circ} \mathrm{C}$ for $30 \mathrm{~min}$ and then stained with crystal violet at room temperature for $30 \mathrm{~min}$. Cells in six randomly selected visual 


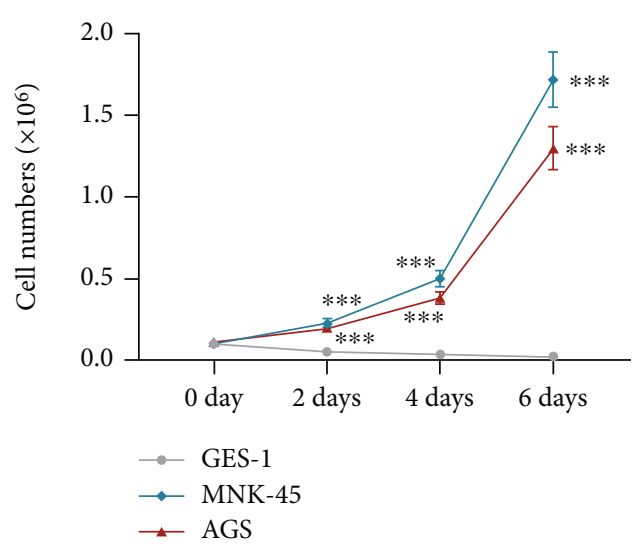

(a)

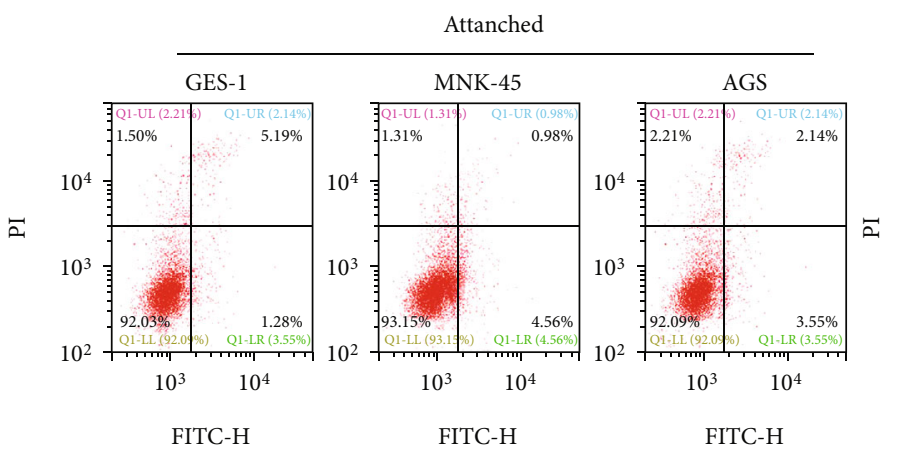

GES-1

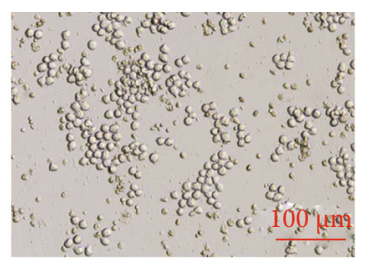

MNK-45

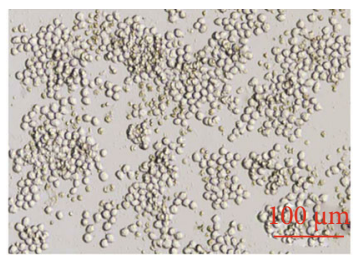

(d)

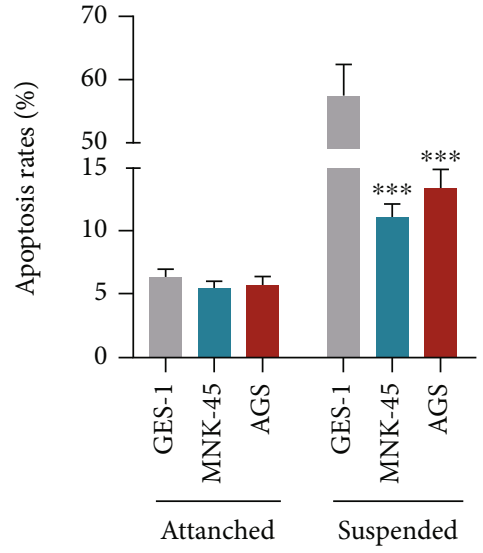

(b)

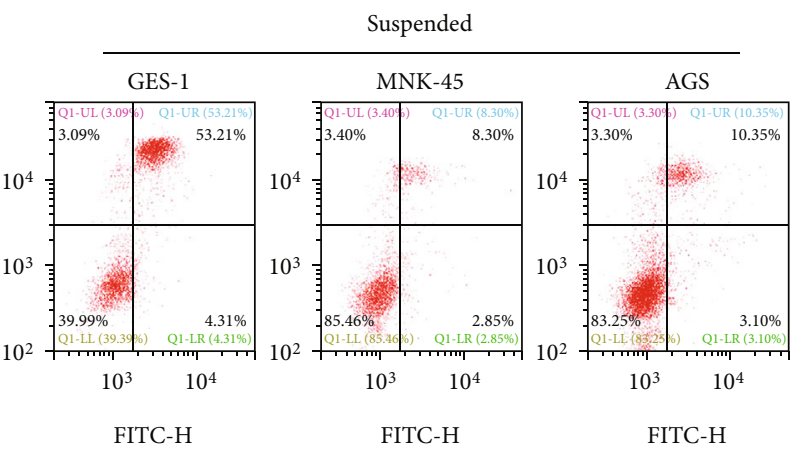

(c)
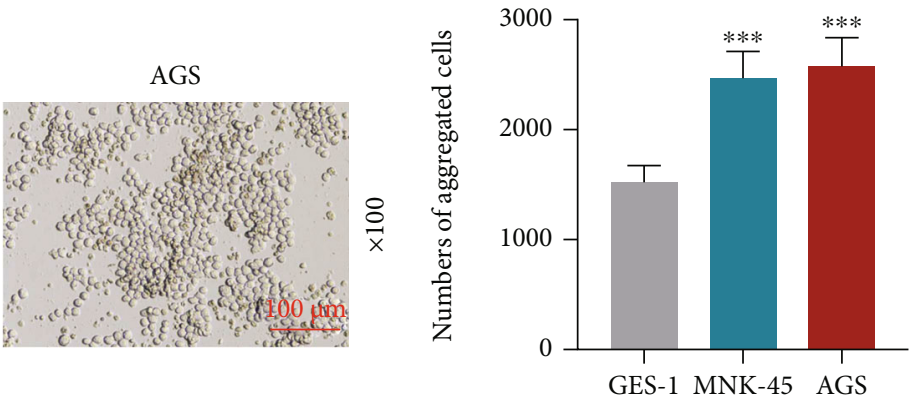

(e)

Figure 1: Continued. 


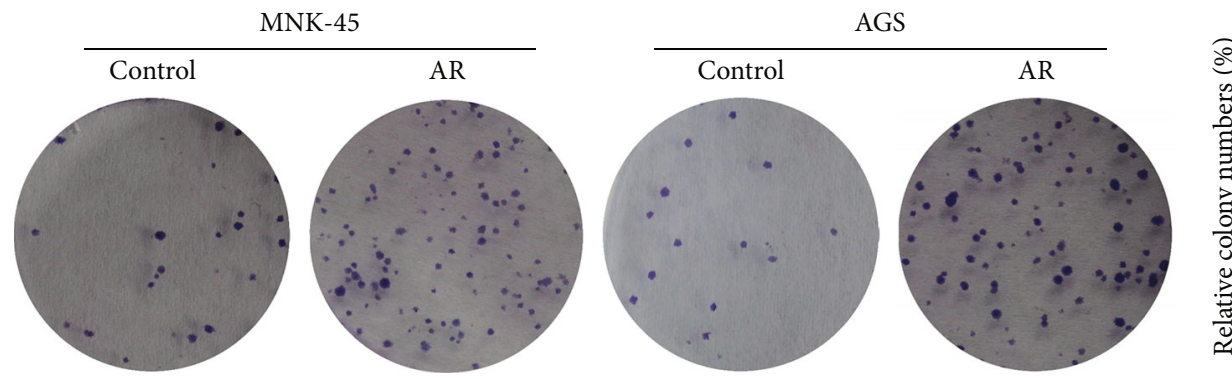

(f)

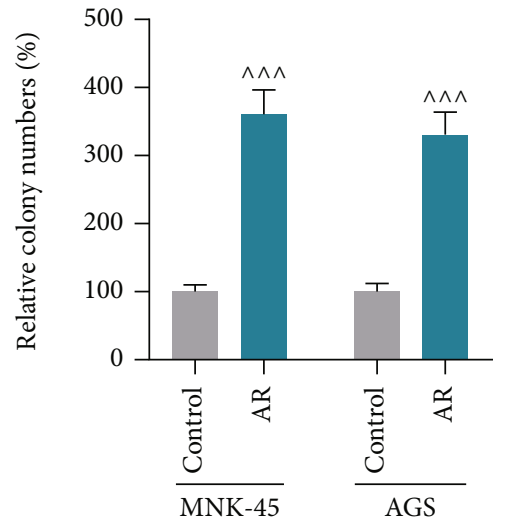

(g)

FIGURE 1: Gastric cancer cells have stronger anoikis resistance compared with normal gastric mucosa epithelial cells. (a) Numbers of GES-1, $M N K-45$ and AGS cells were counted by a hemacytometer after suspension culture for 0, 2, 4, and 6 days. (b) Apoptosis rates of GES-1, MNK45 and AGS cells after 24-hour adherent culture or suspension culture were quantitated through cell apoptosis assay. (c) Cell apoptosis was evaluated using a flow cytometer and an Annexin-V-FITC Apoptosis Detection Kit with PI. (d) Pictures of the morphology of GES-1, MNK45 and $A G S$ cells in suspension culture were taken under a microscope. (e) Numbers of aggregated GES-1, MNK-45 and AGS cells in suspension culture were counted by microscopy. (f) Representative pictures of colonies in colony formation assay. (g) Relative cell colony numbers were counted manually. ${ }^{*} p<0.05$ vs. GES- 1 cells; ${ }^{* * *} p<0.001$ vs. GES- 1 cells and ${ }^{\wedge \wedge} p<0.001$ vs. control group. Data were performed as the means \pm standard.

fields from each room were counted under a NEXCOPENIB-620 inverted biological microscope (magnification: 250x; Chensheng Optical Instrument Technology Co., Ltd., Shenzheng, China).

2.8. Transfection of FASN Silencing RNA. Cells in logarithmic phase were trypsinized, seeded in a 24 -well plate at a density of $5 \times 10^{3}$ cells per well and cultured until $30-50 \%$ confluence. To downregulate the expression of FASN, small interfering RNA targeting FASN (siFASN; siB111124103713-1-5, UGAGAAAGGUCGAAUUUGCCA, Guangzhou RiboBio Co., Ltd, Guangzhou, China) was transfected into the cells. Lipofectamine 2000 (11668019, Thermo Fisher Scientific, Waltham, MA, USA) was used during the $48 \mathrm{~h}$ transfection of siFASN and small interfering RNA of negative control (siNC; siN0000002-1-5, Guangzhou RiboBio Co., Ltd, Guangzhou, China).

\subsection{Quantitative Reverse Transcription-Polymerase Chain} Reaction ( $q R T-P C R)$. Total RNA was extracted from cells using a TRIzol kit (B511321-0100, Sangon Biotech Co., Ltd., Shanghai, China). Reverse transcription was conducted to synthesize cDNA by using a PrimeScript RT Reagent Kit (638314, Takara Bio, Inc., Otsu, Japan). Sequences of the primers (Shanghai GenePharma Co., Ltd., Shanghai, China) were listed in Table 1. Then the cDNA was tracked using SYBR Green qPCR Supermix (1725270, Bio-Rad Laboratories, Inc., CA, USA) through a CFX384 RT-PCR cycler (Bio-Rad Laboratories, Inc., CA, USA) under a thermal cycling program containing 40 cycles of $95^{\circ} \mathrm{C}$ for $3 \mathrm{~min}$, $95^{\circ} \mathrm{C}$ for $15 \mathrm{~s}$, and $60^{\circ} \mathrm{C}$ for $15 \mathrm{~s}$. The expression levels of relative FASN RNA were calculated on the basis of the mean $\beta$-actin expression levels in the representative sample. Data were analyzed using the relative quantification method $2^{-\Delta \Delta C T}[20]$.
2.10. Western Blot. Total protein was extracted from cells using radio immunoprecipitation assay (RIPA) lysis buffer (R0010-100 ml, Beijing Solarbio Science \& Technology Co., Ltd., Beijing, China) with phenylmethanesulfonyl fluoride (PMSF) (P0100-100 ml, Beijing Solarbio Science \& Technology Co., Ltd., Beijing, China), and then the protein was centrifuged at $12,000 \times \mathrm{g}$ at $4^{\circ} \mathrm{C}$ for $10 \mathrm{~min}$ to collect the supernatant. The concentration of total protein was determined by a Bradford assay kit (5000001, Bio-Rad Laboratories, Inc., CA, USA). Equal amounts of protein samples were separated by 10\% SDS (A600485-0100, Sangon Biotech Co., Ltd., Shanghai, China) polyacrylamide gel electrophoresis (SDS-PAGE) and transferred to PVDF membranes (88585, Thermo Fisher Scientific, Waltham, MA, USA). The membranes were blocked in 5\% bovine serum albumin (BSA; E661003-0100, Sangon Biotech Co., Ltd., Shanghai, China) for $1 \mathrm{~h}$ and then incubated overnight at $4^{\circ} \mathrm{C}$ with the antibodies including anti-FASN (ab128870, 1:10000; Abcam, Cambridge, MA, USA), anti-p-ERK1/2 (ab214362, $1: 1000$; Abcam, Cambridge, MA, USA), anti-ERK1/2 (ab184699, 1:10000; Abcam, Cambridge, MA, USA), antiBcl-x (ab32370, 1:1000; Abcam, Cambridge, MA, USA), and anti- $\beta$-actin (ab8226, $1: 1000$; Abcam, Cambridge, MA, USA). The next day, the membranes were removed and washed with Tris-buffered saline containing Tween (TBST; C520009-0001, Sangon Biotech Co., Ltd., Shanghai, China), followed by being incubated with corresponding secondary antibodies conjugated to Horseradish Peroxidase (HRP; ab6721, 1:5000; Abcam, Cambridge, MA, USA) for $1 \mathrm{~h}$ at room temperature and washed five times with TBST for 5 min each time. ECL reagent (32209, Thermo Fisher Scientific, Waltham, MA, USA) was added to the membranes for the visualization in a dark room at room temperature. The protein level was analyzed using Image J software, version 1.48 (National Institutes of Health, Bethesda, MD, USA). 


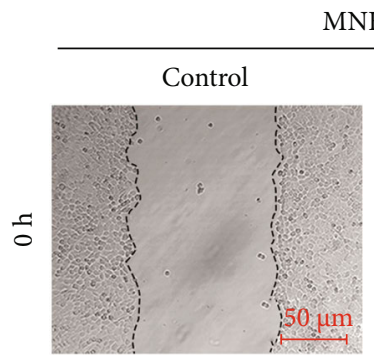

MNK-45

AGS
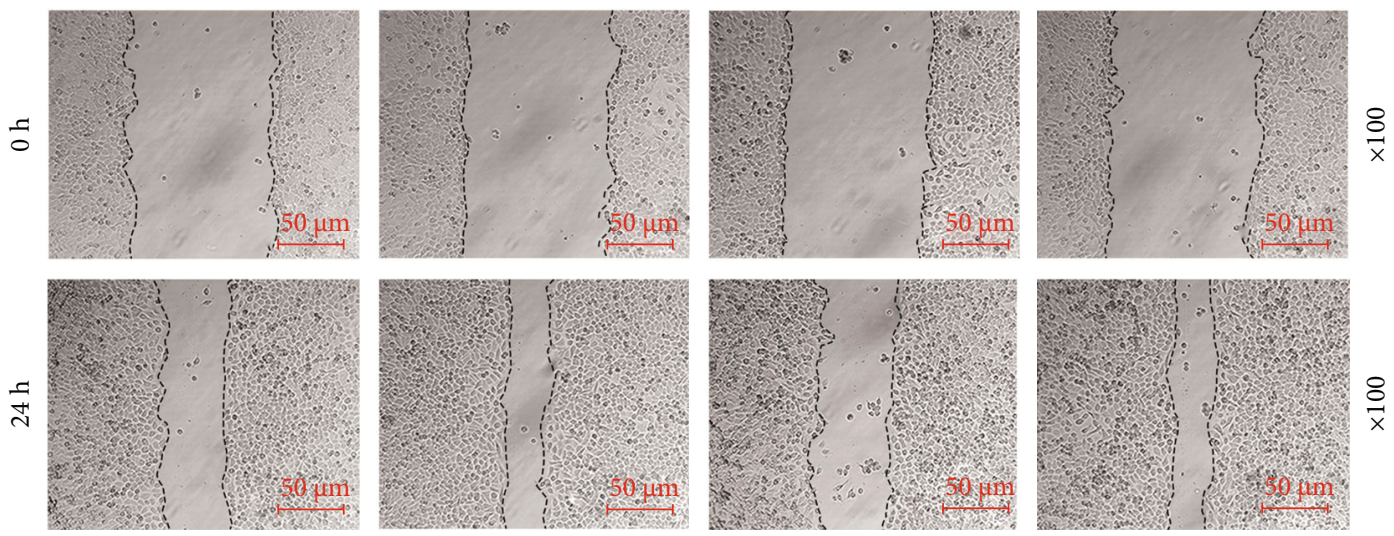

(a)

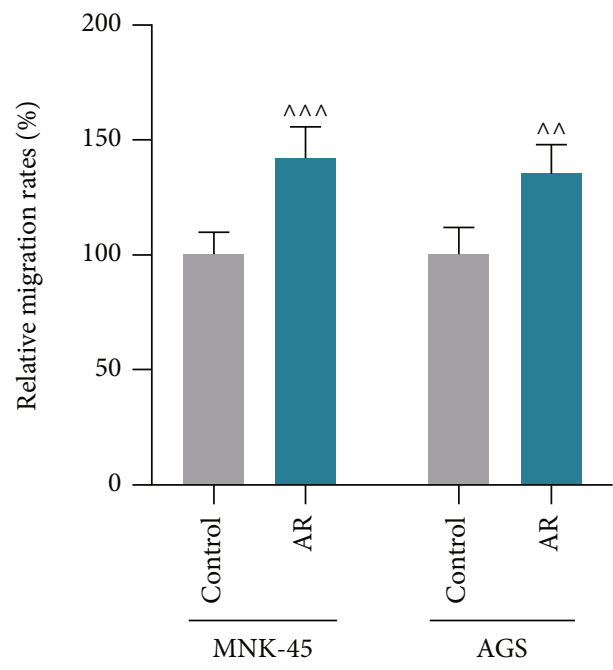

(b)

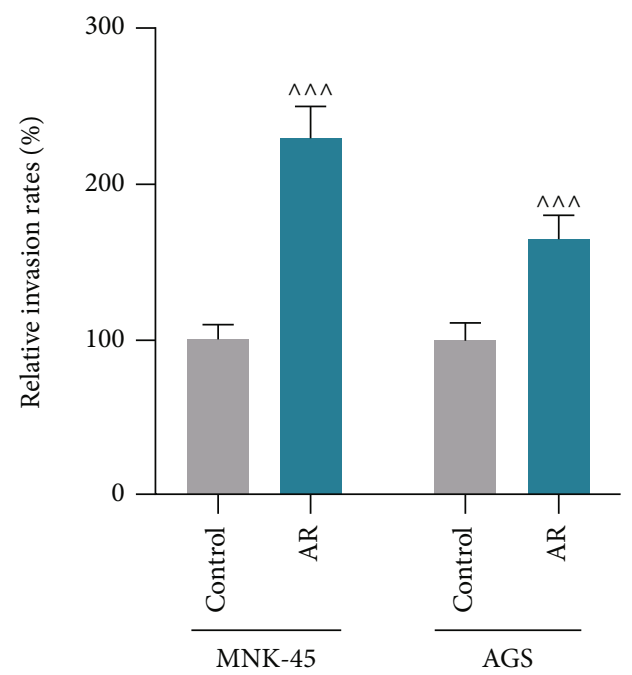

(c)

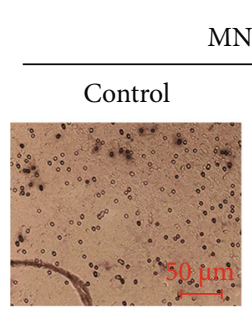

MNK-45

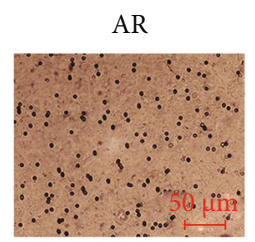

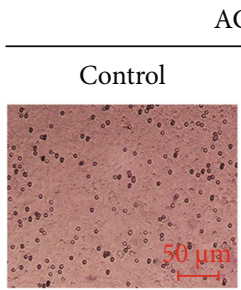

(d)

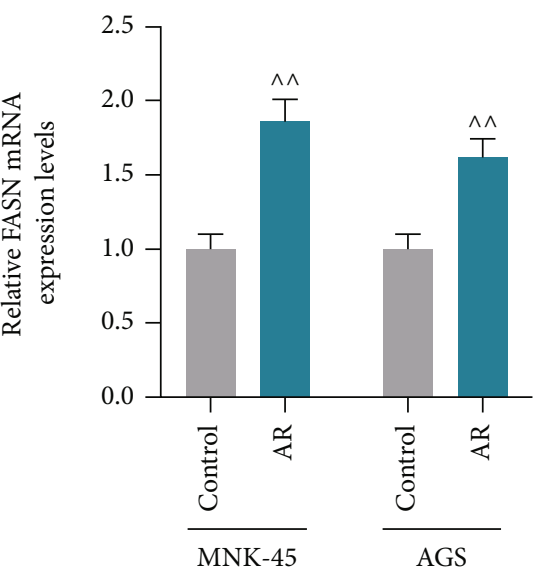

(e)

Figure 2: Continued. 


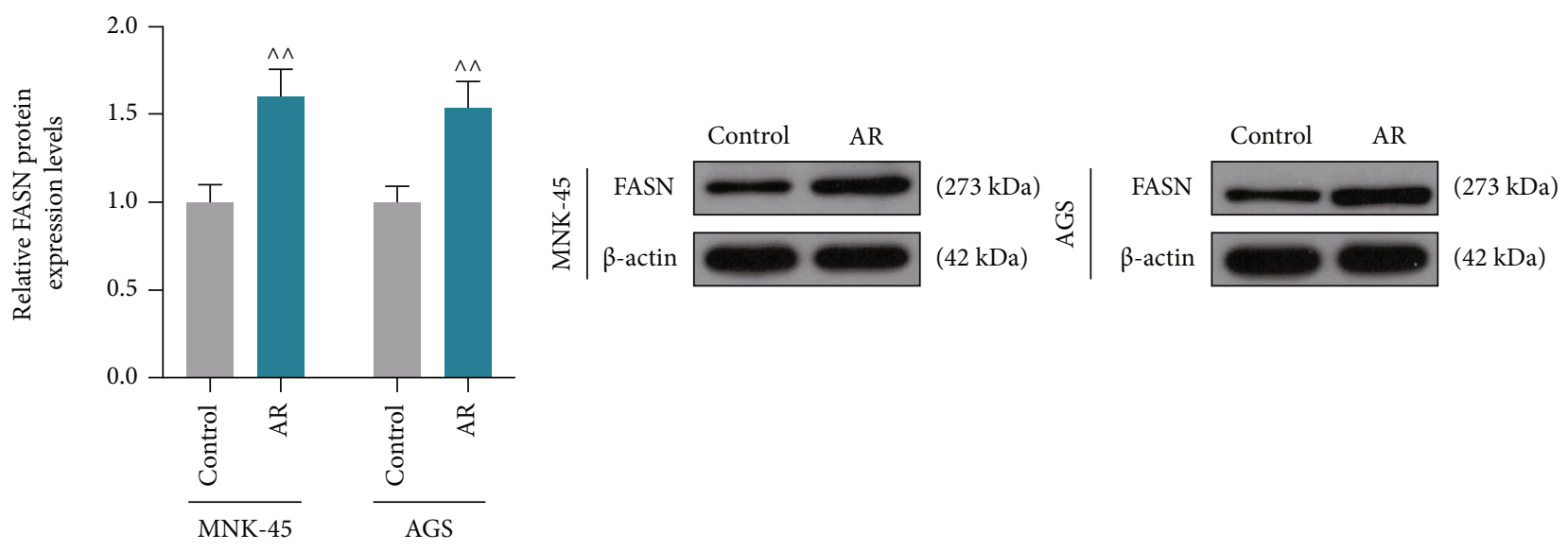

(f)

(g)

FIGURE 2: Anoikis resistance (AR) promoted gastric cancer cell migration and invasion, and fatty acid synthase (FASN) was related to AR. (a) Representative images of the migration of MNK-45 and AGS cell in the AR and control groups determined by wound healing assay. (b) Relative migration rates of $M N K-45$ and $A G S$ cells in the AR and control groups were determined by wound healing assay. (c) Representative images of the invasion of MNK-45 and AGS cell in the AR and Control groups determined by Transwell invasion assay. (d) Relative invasion rates of $M N K-45$ and AGS cells in the AR and control groups were determined by Transwell invasion assay. (e) Relative FASN mRNA expression levels in MNK-45 and AGS cells in the AR and control groups were evaluated by quantitative reverse transcription-polymerase chain reaction (qRT-PCR). (f) Representative pictures of relative FASN protein expression levels in $M N K-45$ and AGS cells in the AR and control groups during western blot. $\beta$-Actin was used as a loading control. (g) Relative FASN protein expression levels in MNK-45 and AGS cells in the AR and control groups were assessed by western blot. Cells under adherent culture were used as the control group and received no treatment. ${ }^{\wedge \wedge} p<0.01$ vs. control group; ${ }^{\wedge \wedge} p<0.001$ vs. control group. Data were performed as the means \pm standard.

2.11. Statistical Analysis. GraphPad Prism 8.0 (GraphPad Software Inc., San Diego, CA, USA) and SPSS 20.0 software (SPSS Inc., Chicago, IL, USA) were used to conduct the statistical analysis. Data were performed as the means \pm standard. The statistical significance of differences between two groups was determined by independent-samples $t$-test. Comparisons among more than two groups were conducted using one-way ANOVA followed by Tukey's post hoc test. $\mathrm{P}<0.05$ was considered to be a statistically significant difference.

\section{Results}

3.1. GC Cells Showed Stronger AR Compared with Normal Gastric Mucosa Epithelial Cells. During the culture in suspension for 2, 4 and 6 days, the number of GES- 1 cells decreased while those of $M N K-45$ and $A G S$ cells increased significantly (Figure 1(a), $p<0.001$ ). GES-1, MNK-45 and AGS cells were all attached cells, some of which died during the culture in suspension, and we found the apoptosis rate of the GES-1 cells in suspension was higher than the attached GES-1 cells. And the apoptosis rate of normal gastric mucosa epithelial cells was higher than GC cells in both attached and suspended condition (Figures 1(b), 1(c), $p<0.05, p<0.001$ ). In suspension culture, a multicellular aggregate is more resistant to anoikis than single suspension cells [21]. As suggested by the results of the morphology on the cell, which was observed by microscopy,, MNK-45 and AGS cells formed larger clusters and more aggregated cells when compared with GES-1 cells (Figures $1(\mathrm{~d}), 1(\mathrm{e}), p<0.001$ ).
3.2. AR Promoted GC Cell Multiplication, Migration and Invasion and FASN Was Associated with AR. In the results of colony formation assay, it was presented that the colony numbers of MNK-45 and AGS cells in the AR group were greater than those in the control group, indicating that cells with AR were more capable of proliferation (Figures 1(f), $1(\mathrm{~g}), p<0.001)$. During the wound healing assay and Transwell invasion assay, both the migration and invasion rates of $M N K-45$ and AGS cells were higher in the AR group than in the control group (Figures $2(\mathrm{a})-2(\mathrm{~d}), p<0.01, p<0.001$ ). We also found that the relative mRNA and protein levels of FASN were higher in MNK-45 and AGS cells of the AR group as compared with those of the control group, which suggested an association between FASN and AR (Figures 2(e)$2(\mathrm{~g}), p<0.01, p<0.001)$.

3.3. SiFASN Promoted Apoptosis Yet Suppressed FASN Expression, Aggregation, Migration and Invasion in GC Cells. Both the relative mRNA and protein expression levels of FASN in MNK-45 and AGS cells were lower in the AR + siFASN group than in the AR + siNC group (Figures $3(\mathrm{a})-$ $3(c), p<0.001)$, implying that transfection of siFASN suppressed the expression of FASN. Silence of FASN promoted the apoptosis rates of $M N K-45$ and AGS cells while decreasing the number of aggregated cells. (Figures $3(\mathrm{~d})-3(\mathrm{~g}), p<$ $0.01, P<0.001)$. In addition, the relative migration and invasion rates of MNK-45 and AGS cells in the AR+siFASN group reduced significantly in comparison with the $A R$ + siNC group (Figures 4(a) $-4(\mathrm{~d}), p<0.001$ ). 


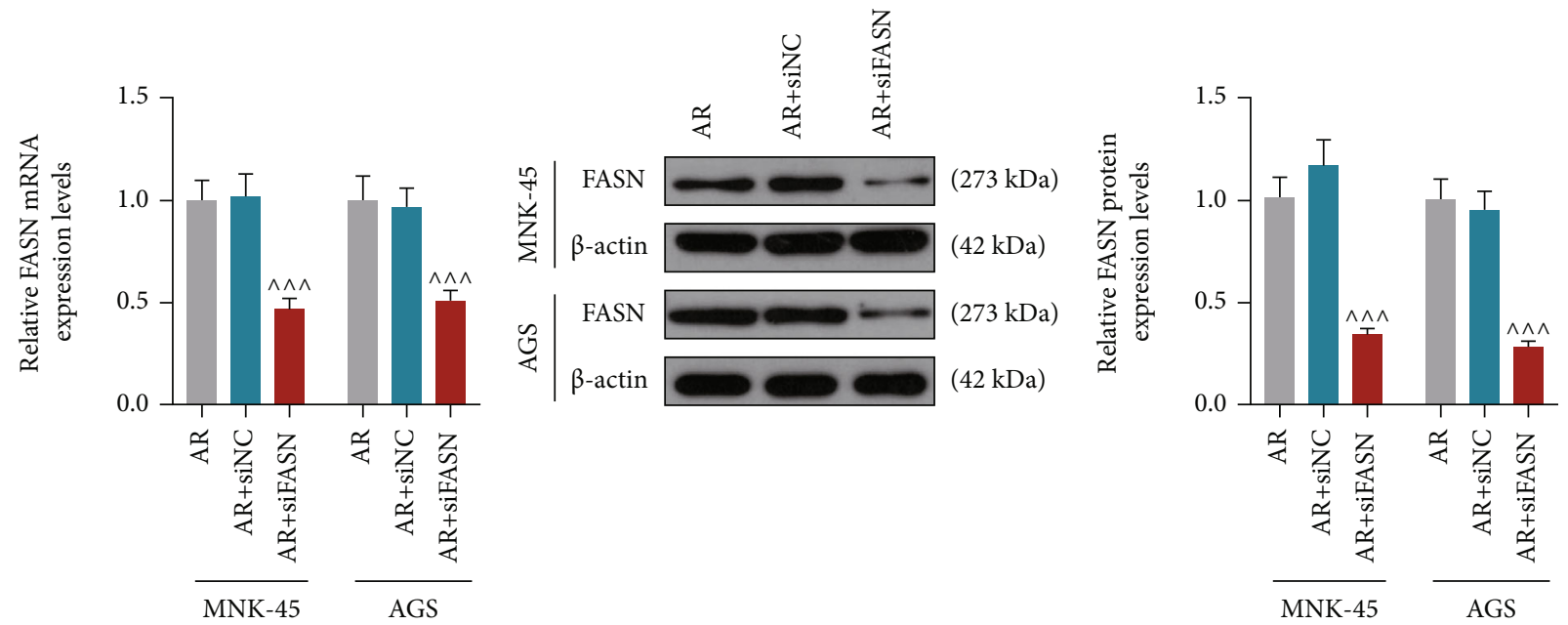

(a)

(b)

(c)
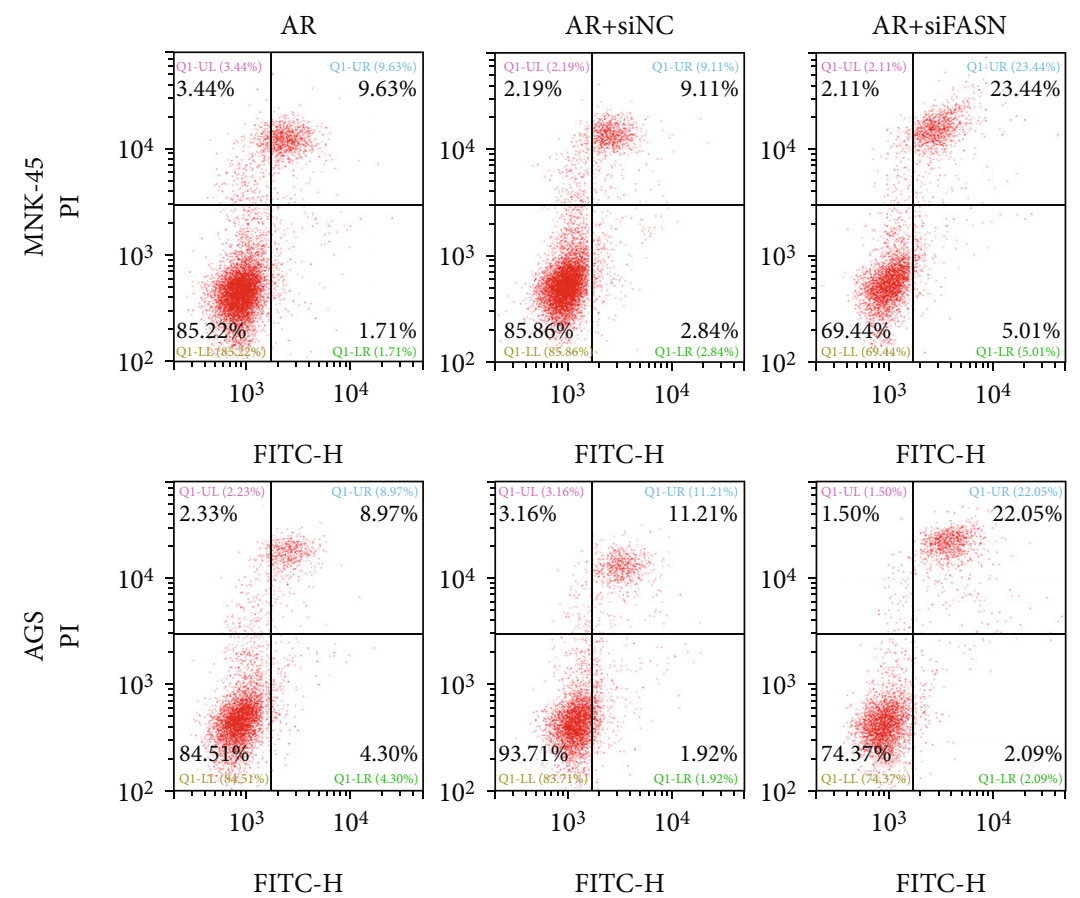

(d)

FIgUre 3: Continued. 


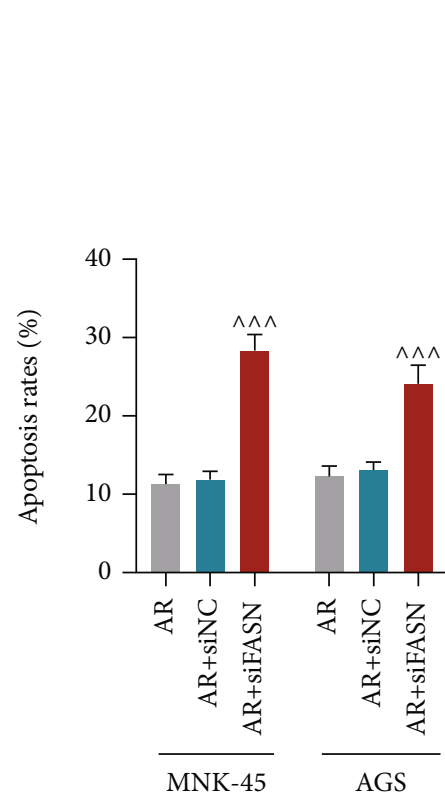

(e)
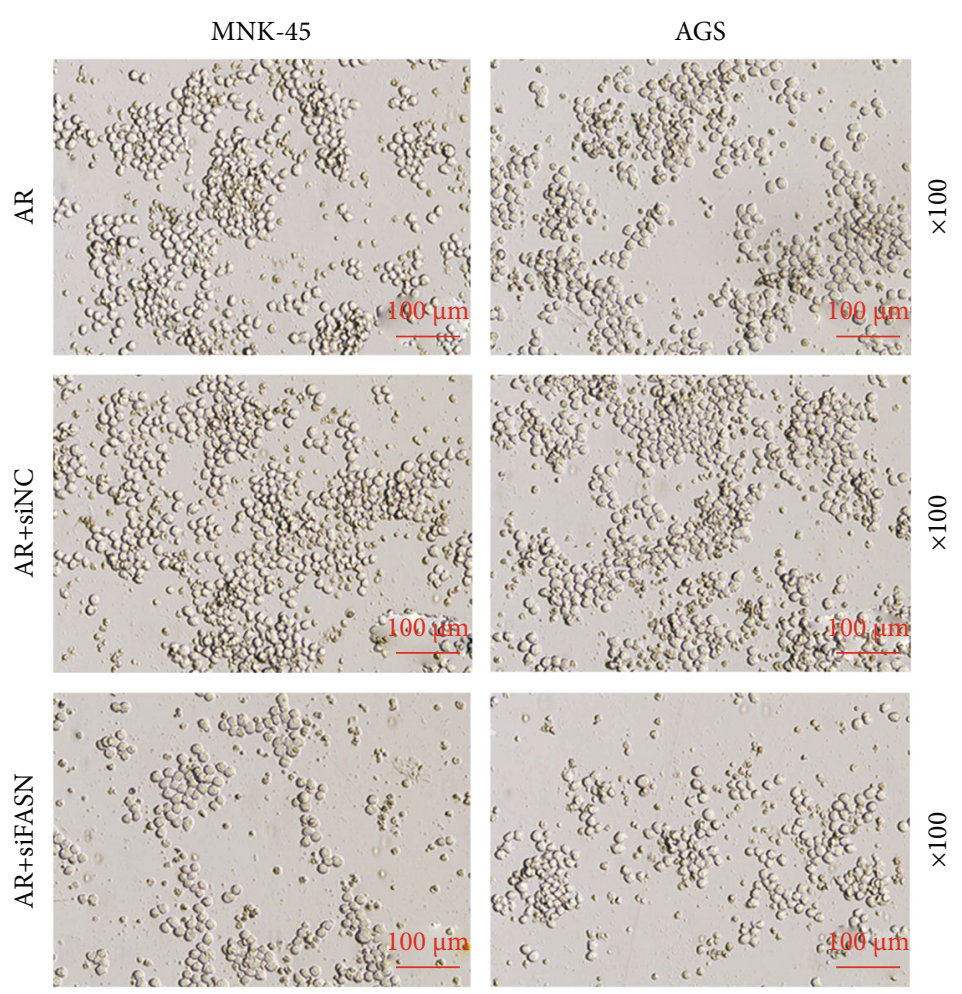

(f)

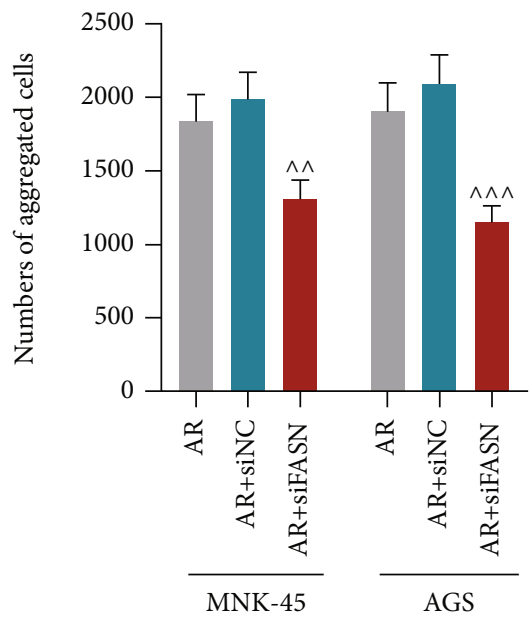

(g)

FIGURE 3: Small interfering RNA targeting fatty acid synthase (siFASN) promoted apoptosis while suppressing cell aggregation and FASN expression. (a) Relative fatty acid synthase (FASN) mRNA expression levels in MNK-45 and AGS cells in the anoikis resistance (AR), AR+ small interfering RNA negative control (siNC), and AR + siFASN groups were evaluated by quantitative reverse transcription-polymerase chain reaction (qRT-PCR). (b) Representative pictures of relative FASN protein expression levels in $M N K-45$ and AGS cells in the AR, AR + siNC, and AR + siFASN groups during western blot. $\beta$-Actin was used as a loading control. (c) Relative FASN protein expression levels in MNK-45 and AGS cells in the AR, AR + siNC, and AR + siFASN groups were assessed by western blot. (d) Cell apoptosis was evaluated using a flow cytometer and an Annexin-V-FITC Apoptosis Detection Kit with PI. (e) Quantitation of the apoptosis rates of MNK-45 and AGS cells in the AR, AR + siNC, AR + siFASN groups determined through cell apoptosis assay. (f) Pictures of the morphology of MNK-45 and $A G S$ cells in the $\mathrm{AR}, \mathrm{AR}+\mathrm{siNC}$, and $\mathrm{AR}+\mathrm{siFASN}$ groups were taken under a microscope during suspension culture. (g) Numbers of aggregated $M N K-45$ and $A G S$ cells in the $\mathrm{AR}, \mathrm{AR}+\mathrm{siNC}$, and $\mathrm{AR}+\operatorname{siFASN}$ groups were manually counted by microscopy. ${ }^{\wedge \wedge} p<0.01$ vs. $\mathrm{AR}+$ siNC group; ${ }^{\wedge \wedge} p<0.001$ vs. $\mathrm{AR}+$ siNC group. Data were performed as the means \pm standard.

3.4. SiFASN Significantly Suppressed the Activation of PERK1/2/Bcl-xL Pathway in GC Cells with AR. The relative protein expression levels of p-ERK1/2 and Bcl-xl in $M N K-45$ and $A G S$ cells in the AR+siFASN group was detected to be declined while the expression of ERK1/2 was basically unchanged (Figures 5(a), 5(b), $p<0.05, P<0.01, P<0.001$ ), leading to the lowest ratio of $\mathrm{p}$-ERK1/2 to ERK1/2 in the $\mathrm{AR}+\operatorname{siFASN}$ group (Figure $5(\mathrm{c}), p<0.01, P<0.001$ ). These 


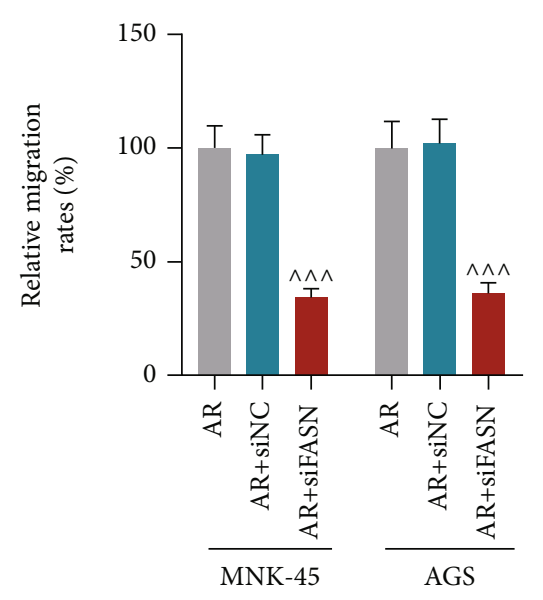

(a)

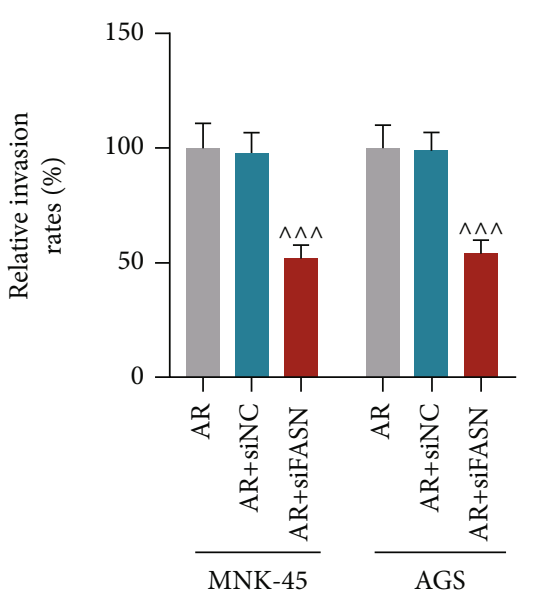

(b)
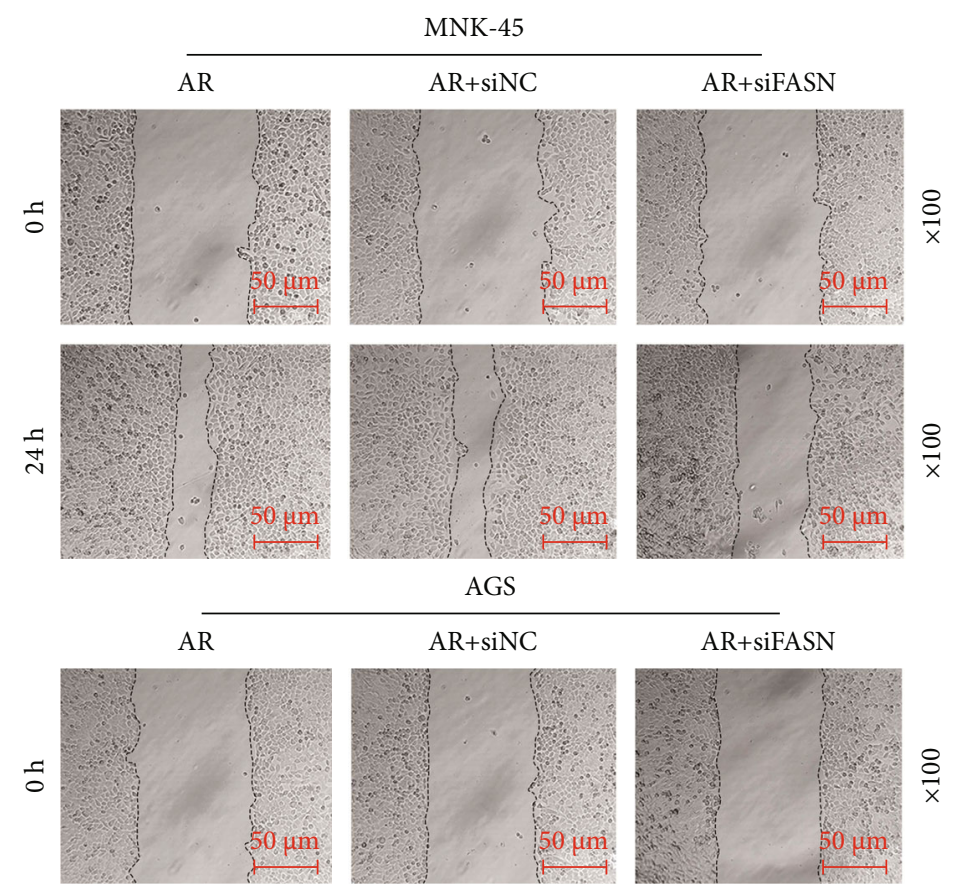

$\mathrm{AR}+\mathrm{siNC}$

AR+siFASN
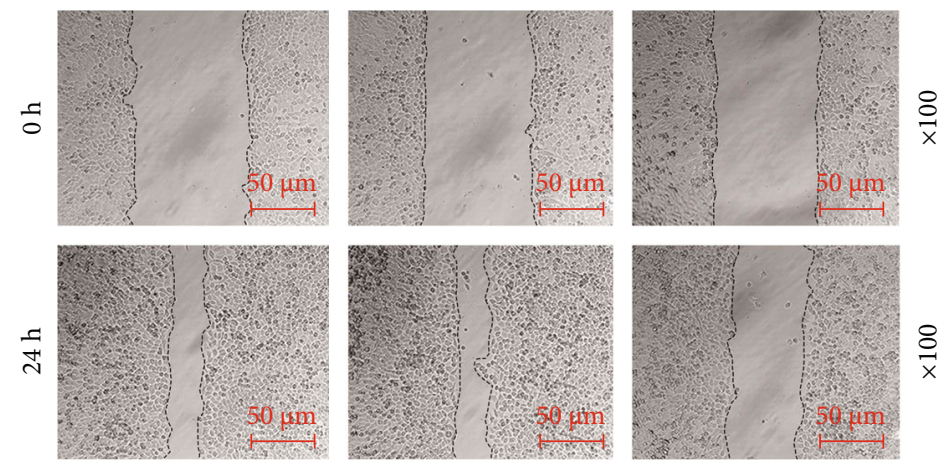

(c)

Figure 4: Continued. 


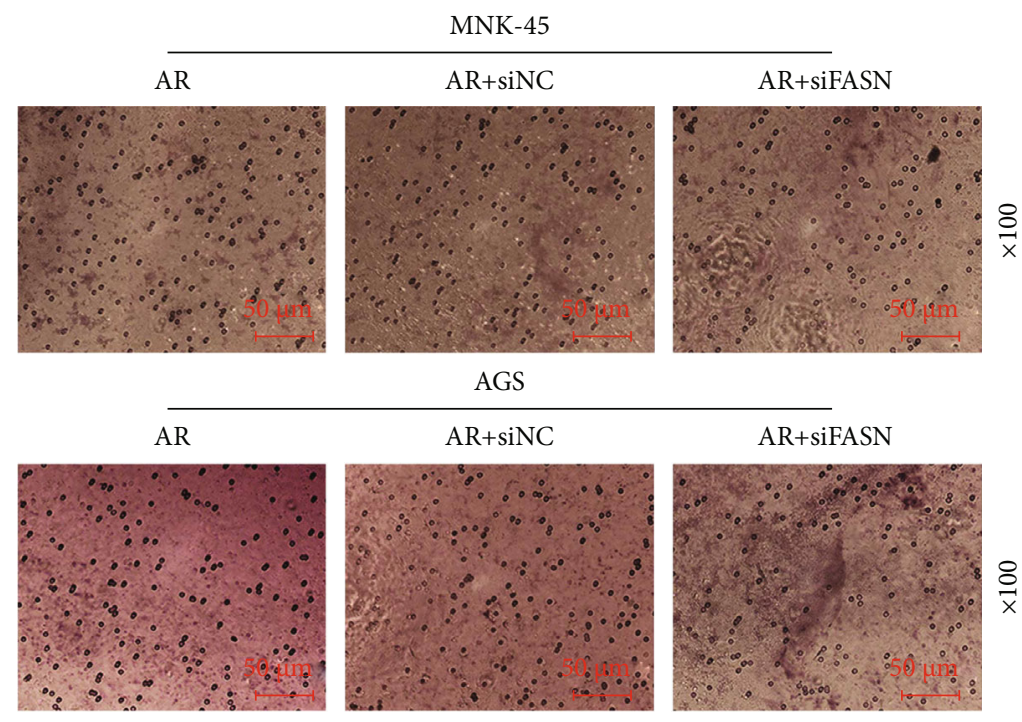

(d)

FIGURE 4: Small interfering RNA targeting fatty acid synthase (siFASN) suppressed cell migration and invasion. (a) Relative migration rates of $M N K-45$ and $A G S$ cells in the anoikis resistance (AR), AR + small interfering RNA negative control (siNC), and AR + siFASN groups were determined by wound healing assay. (b) Relative invasion rates of MNK-45 and AGS cells in the AR, AR+ siNC, and AR + siFASN groups were determined by transwell invasion assay. (c) Representative images of the migration of $M N K-45$ and AGS cell migration in the AR, $\mathrm{AR}+\mathrm{siNC}$, and AR + siFASN groups determined by wound healing assay. (d) Representative images of the invasion of $M N K-45$ and $A G S$ cell invasion in the AR, AR + siNC, and AR + siFASN groups determined by transwell invasion assay. ${ }^{\wedge \wedge} p<0.001 \mathrm{vs.} \mathrm{AR+siNC} \mathrm{group}$. Data were performed as the means \pm standard.

observations suggested that the silence of FASN inhibited the activation of $\mathrm{p}$-ERK1/2/Bcl-xL pathway, and FASN/pERK1/2/Bcl-xL pathway might play vital roles in GC with AR.

\section{Discussion}

GC is a kind of deadly malignancy with a low early diagnosis rate and unfavorable prognosis. Tumor metastasis is a major reason for the failure in the treatment and mortality in patients in nearly all types of cancer, and was evidenced in approximately $50 \%$ of patients during the development of tumor at initial diagnosis [22, 23]. Cancer metastasis is a complex process comprising different stages, including local invasion, intravasation, survival in circulation, extravasation, and colonization and metastasis, in which a number of interactions between tumor and stroma have been reported [24, 25]. Anoikis, a programmed cell death induced upon the detachment of cells from ECM, is deemed as a crucial mechanism in the preclusion on adherent-independent cell growth and the attachment to an improper matrix, which thereby prevents the metastasis and colonization of cells in distant organs [26]. In addition, Anoikis is one of key procedures to maintain the normal development and homeostasis of organisms [27]. AR, the capability of cells to survive during the detachment from the ECM, is a prerequisite for the development of tumor metastasis [28]. Thus, the competence of cancer cells to resist anoikis has attracted the increasing attention from the scientific community in the field of cancer research, and so far, several mechanisms of AR have been reported and proposed. For instance, integrin- $\alpha 2 \beta 1 /-\alpha 5 \beta 1$ interacted with epidermal growth factor receptor (EGFR) in the AR of colon cancer [29]. MiR-141/KLF12/Sp1/survivin signaling pathway enhanced $\mathrm{AR}$ in ovarian cancer, and a transcriptomic study addressed the importance of CDKN1A-regulated quiescence for cells to survive from anoikis in head and neck squamous carcinoma [30, 31]. In accordance with the discoveries in previous studies, FASN, an enzyme implicated in the process of metabolism, has shown to be positively correlated with the development of various cancers, including GC [32-34]. FASN has also been proved to mediate AR during cell growth and metastasis in osteosarcoma. However, the role of FASN in GC cells with AR has not been explored in detail [19].

In the present study, the observations suggested that AR promoted cell multiplication, migration and invasion and led to high expression levels of FASN in cells, which indicated a possible relation between FASN and AR. We also discovered that silence of FASN significantly reduced cell multiplication, migration and invasion as well as p-ERK1/2 and Bcl$\mathrm{xL}$ levels while barely affecting the expression of ERK1/2. PERK1/2 may serve as an important hub in the network, as it occurs crosstalk with other pathways, including phosphatidylinositol 3-kinase (PI3K) [35], and Wnt [36]. Moreover, it has also been demonstrated that there exists a regulatory loop between FASN and ERK pathway, in which PI3K/AKT pathway is involved as well [37]. Therefore, the regulation between FASN and ERK pathway may be involved with other molecular pathways. PI3K/AKT pathway also regulates downstream anti-apoptotic proteins, including $\mathrm{Bcl}-\mathrm{xL}$ [38]. Thus, it was inferred that the functions of FASN on GC cells with AR might be related to $\mathrm{p}-\mathrm{ERK} 1 / 2 / \mathrm{Bcl}-\mathrm{xL}$ pathway, regardless of other molecular pathways that may be involved. 


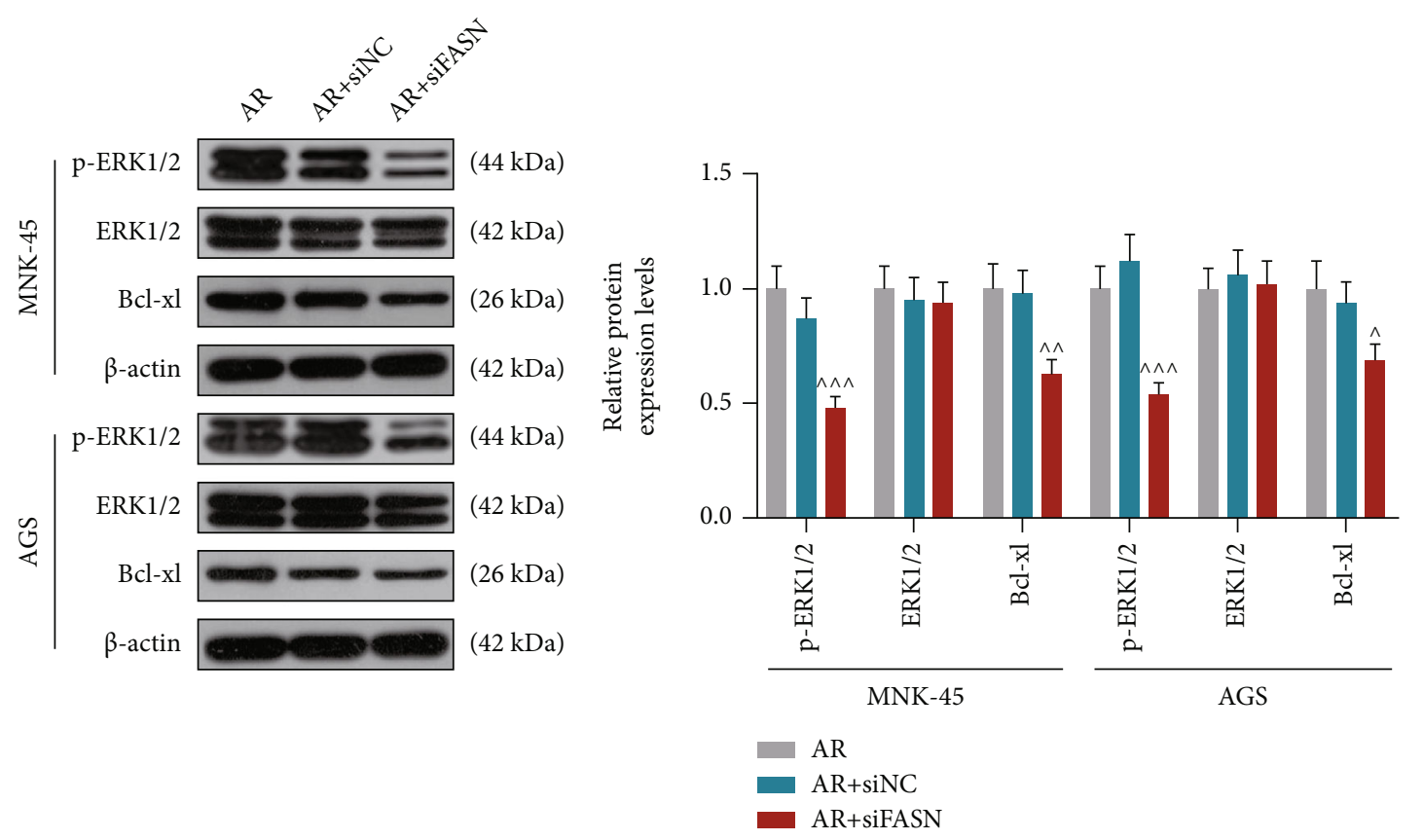

(a)

(b)

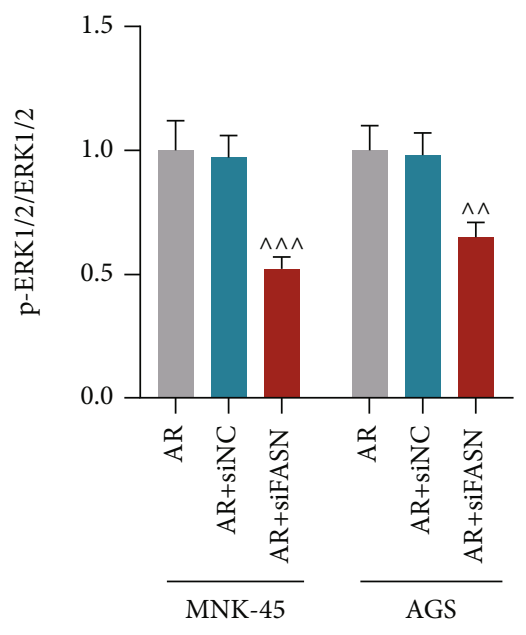

(c)

FIGURE 5: Small interfering RNA targeting fatty acid synthase (siFASN) significantly suppressed p-ERK1/2/Bcl-xL pathway activation in anoikis resistant gastric cancer cells. (a) Representative pictures of the protein levels of phosphorylated (p)-ERK1/2, ERK1/2 and Bcl-xL in $M N K-45$ and $A G S$ cells in the anoikis resistance (AR), AR + small interfering RNA negative control (siNC), AR + siFASN groups during western blot. $\beta$-Actin was used as a loading control. (b) Quantitative analysis of p-ERK1/2, ERK1/2 and Bcl-xL expressions normalized to $\beta$-actin through western blot. (c) The Relative ratio of $\mathrm{p}$-ERK1/2 to ERK1/2 expression. ${ }^{\wedge} p<0.05$ vs. AR $+\operatorname{siNC}$ group; ${ }^{\wedge \wedge} p<0.01$ vs. AR + siNC group; ${ }^{\wedge \wedge} p<0.001$ vs. AR + siNC. Data were performed as the means \pm standard.

However, there is room for improvement of the current study. Firstly, the length of FASN mRNA is about $8.4 \mathrm{~kb}$, making the clone on the full length a great challenge. Thus, cell lines with overexpressed FASN failed to be established, leaving the results and conclusions with one-sided uncertainty. Also, the specific molecular mechanism of FASN in GC cells with AR was not absolutely settled. Secondly, the experiments for detecting cell apoptosis rates and colony formation could be conducted more scrupulously by the exclusion of the influence of cell proliferation. Thirdly, we directly selected $\mathrm{p}$-ERK1/2/Bcl-xL based on documents and assumption, and this blindness could be avoided by probing into the downstream signaling pathways of FASN. Lastly, researches in vivo should be carried out in the future to further prove the conclusions of experiments in vitro.

In summary, this study demonstrated the stronger AR of GC cells, which may explain the survival of cells during the metastasis and spread of GC. The results revealed that AR facilitated multiplication, migration and invasion while impeding apoptosis of GC cell. Moreover, it was preliminarily verified that the down-regulation of FASN exerted suppressive effects on GC cells with $A R$, which was associated with the inhibition of $\mathrm{p}-\mathrm{ERK} 1 / 2 / \mathrm{Bcl}-\mathrm{xL}$ signaling pathway. Taken together, our results and data indicate that FASN 
may become a new target for preventing the metastasis of tumor, thus providing a novel reference for the treatment against cancer. Nonetheless, more researches and clinical trials are needed to explore whether FASN is a feasible mean for the prevention and treatment of GC.

\section{Data Availability}

The analyzed data sets generated during the study are available from the corresponding author on reasonable request.

\section{Conflicts of Interest}

The authors declare no conflicts of interest.

\section{Acknowledgments}

This work was supported by Anhui Province Nature Science Foundation [1808085MH305]; National Innovation and Entrepreneurship Training Program for College Students [201810367029]; Natural Science Research Projects of Colleges and Universities in Anhui Province [KJ2019A0330].

\section{References}

[1] J. Ferlay, I. Soerjomataram, R. Dikshit et al., "Cancer incidence and mortality worldwide: sources, methods and major patterns in GLOBOCAN 2012," International journal of cancer, vol. 136, no. 5, pp. E359-E386, 2015.

[2] R. B. Hamanaka and N. S. Chandel, "Cell biology. Warburg effect and redox balance," Science, vol. 334, no. 6060, pp. 1219-1220, 2011.

[3] S. P. Hussain, L. J. Hofseth, and C. C. Harris, "Radical causes of cancer," Nature reviews Cancer, vol. 3, no. 4, pp. 276-285, 2003.

[4] Z. Cao, T. Livas, and N. Kyprianou, "Anoikis and EMT: lethal "liaisons" during Cancer progression," Critical reviews in oncogenesis, vol. 21, no. 3-4, pp. 155-168, 2016.

[5] S. J. Strauss, T. Ng, A. Mendoza-Naranjo, J. Whelan, and P. H. Sorensen, "Understanding micrometastatic disease and Anoikis resistance in Ewing family of tumors and osteosarcoma," The oncologist, vol. 15, no. 6, pp. 627-635, 2010.

[6] A. Baron, T. Migita, D. Tang, and M. Loda, "Fatty acid synthase: a metabolic oncogene in prostate cancer?," Journal of Cellular Biochemistry, vol. 91, no. 1, pp. 47-53, 2004.

[7] Y. Jiang, X. Yin, L. Wu, Q. Qin, and J. Xu, "MAPK/P53-mediated FASN expression in bone tumors," Oncology letters, vol. 13, no. 6, pp. 4035-4038, 2017.

[8] T. Migita, S. Ruiz, A. Fornari et al., "Fatty acid synthase: a metabolic enzyme and candidate oncogene in prostate cancer," Journal of the National Cancer Institute, vol. 101, no. 7, pp. 519-532, 2009.

[9] A. Schcolnik-Cabrera, A. Chávez-Blanco, G. DomínguezGómez et al., "Orlistat as a FASN inhibitor and multitargeted agent for cancer therapy," Expert opinion on investigational drugs, vol. 27, no. 5, pp. 475-489, 2018.

[10] L. Sun, Y. Yao, G. Pan et al., "Small interfering RNA-mediated knockdown of fatty acid synthase attenuates the proliferation and metastasis of human gastric cancer cells via the mTOR/Gli1 signaling pathway," Oncology letters, vol. 16, no. 1, pp. 594-602, 2018.
[11] N. Hu, Y. Li, Y. Zhao et al., "A novel positive feedback loop involving FASN/p-ERK1/2/5-LOX/LTB4/FASN sustains high growth of breast cancer cells," Acta Pharmacologica Sinica, vol. 32, no. 7, pp. 921-929, 2011.

[12] T. Puig, H. Aguilar, S. Cufí et al., "A novel inhibitor of fatty acid synthase shows activity against HER2+ breast cancer xenografts and is active in anti-HER2 drug-resistant cell lines," Breast cancer research : BCR, vol. 13, no. 6, p. R131, 2011.

[13] A. P. de Sousa Mesquita, L. S. de Araújo, P. C. A. Pernambuco Filho, H. B. Nader, and C. C. Lopes, "Acquisition of anoikis resistance promotes alterations in the Ras/ERK and PI3K/Akt signaling pathways and matrix remodeling in endothelial cells," Apoptosis : an international journal on programmed cell death, vol. 22, no. 9, pp. 1116-1137, 2017.

[14] Y. J. Guo, W. W. Pan, S. B. Liu, Z. F. Shen, Y. Xu, and L. L. Hu, "ERK/MAPK signalling pathway and tumorigenesis," Experimental and therapeutic medicine, vol. 19, no. 3, pp. 19972007, 2020.

[15] F. Liu, X. Yang, M. Geng, and M. Huang, "Targeting ERK, an Achilles' heel of the MAPK pathway, in cancer therapy," Acta pharmaceutica Sinica B, vol. 8, no. 4, pp. 552-562, 2018.

[16] S. S. Gayle, J. M. Sahni, B. M. Webb et al., "Targeting BCL-xL improves the efficacy of bromodomain and extra-terminal protein inhibitors in triple-negative breast cancer by eliciting the death of senescent cells," The Journal of biological chemistry, vol. 294, no. 3, pp. 875-886, 2019.

[17] S. Heimer, G. Knoll, C. Steixner, D. N. Calance, D. T. Trinh, and M. Ehrenschwender, "Hypertonicity-imposed BCL-XL addiction primes colorectal cancer cells for death," Cancer letters, vol. 435, pp. 23-31, 2018.

[18] A. L. Scherr, G. Gdynia, M. Salou et al., "Bcl- $\mathrm{x}_{\mathrm{L}}$ is an oncogenic driver in colorectal cancer," Cell death \& disease, vol. 7, no. 8, article e2342, 2016.

[19] T. Sun, X. Zhong, H. Song et al., “Anoikis resistant mediated by FASN promoted growth and metastasis of osteosarcoma," Cell death \& disease, vol. 10, no. 4, p. 298, 2019.

[20] K. J. Livak and T. D. Schmittgen, "Analysis of Relative Gene Expression Data Using Real-Time Quantitative PCR and the $2^{-\Delta \Delta_{-} C_{-}}$Method," Methods, vol. 25, no. 4, pp. 402-408, 2001.

[21] H. Kita, Y. Hikichi, K. Hikami et al., "Differential gene expression between flat adenoma and normal mucosa in the colon in a microarray analysis," Journal of gastroenterology, vol. 41, no. 11, pp. 1053-1063, 2006.

[22] L. A. Dudjak, "Cancer metastasis," Seminars in oncology nursing, vol. 8, no. 1, pp. 40-50, 1992.

[23] P. S. Steeg, "Targeting metastasis," Nature reviews Cancer, vol. 16, no. 4, pp. 201-218, 2016.

[24] J. Robert, "Biology of cancer metastasis," Bulletin du cancer, vol. 100, no. 4, pp. 333-342, 2013.

[25] R. Zeeshan and Z. Mutahir, "Cancer metastasis - tricks of the trade," Bosnian journal of basic medical sciences, vol. 17, no. 3, pp. 172-182, 2017.

[26] P. Paoli, E. Giannoni, and P. Chiarugi, “_Anoikis_ molecular pathways and its role in cancer progression," Biochimica et Biophysica Acta, vol. 1833, no. 12, pp. 3481-3498, 2013.

[27] E. Kakavandi, R. Shahbahrami, H. Goudarzi, G. Eslami, and E. Faghihloo, "Anoikis resistance and oncoviruses," Journal of Cellular Biochemistry, vol. 119, no. 3, pp. 2484-2491, 2018.

[28] Y. N. Kim, K. H. Koo, J. Y. Sung, U. J. Yun, and H. Kim, “Anoikis resistance: an essential prerequisite for tumor metastasis," 
International journal of cell biology, vol. 2012, Article ID 306879, 11 pages, 2012.

[29] D. Guha, T. Saha, S. Bose et al., "Integrin-EGFR interaction regulates anoikis resistance in colon cancer cells," Apoptosis : an international journal on programmed cell death, vol. 24, no. 11-12, pp. 958-971, 2019.

[30] C. Guo, L. F. Xu, H. M. Li et al., "Transcriptomic study of the mechanism of anoikis resistance in head and neck squamous carcinoma," PeerJ, vol. 7, article e6978, 2019.

[31] C. S. Mak, M. M. Yung, L. M. Hui et al., "MicroRNA-141 enhances anoikis resistance in metastatic progression of ovarian cancer through targeting KLF12/Sp1/survivin axis," Molecular cancer, vol. 16, no. 1, p. 11, 2017.

[32] S. R. Dong, X. L. Ju, and W. Z. Yang, "STAT5A reprograms fatty acid metabolism and promotes tumorigenesis of gastric cancer cells," European review for medical and pharmacological sciences, vol. 23, no. 19, pp. 8360-8370, 2019.

[33] J. Duan, L. Chen, M. Zhou et al., "MACC1 decreases the chemosensitivity of gastric cancer cells to oxaliplatin by regulating FASN expression," Oncology reports, vol. 37, no. 5, pp. 25832592, 2017.

[34] R. Ezzeddini, M. Taghikhani, M. H. Somi, N. Samadi, and M. J. Rasaee, "Clinical importance of FASN in relation to HIF- $1 \alpha$ and SREBP-1c in gastric adenocarcinoma," Life sciences, vol. 224, pp. 169-176, 2019.

[35] E. W. Bradley, M. M. Ruan, A. Vrable, and M. J. Oursler, "Pathway crosstalk between Ras/Raf and PI3K in promotion of M-CSF-induced MEK/ERK-mediated osteoclast survival," Journal of Cellular Biochemistry, vol. 104, no. 4, pp. 14391451, 2008.

[36] T. Hu and C. Li, "Convergence between Wnt- $\beta$-catenin and EGFR signaling in cancer," Molecular cancer, vol. 9, p. 236, 2010.

[37] A. Vazquez-Martin, R. Colomer, J. Brunet, R. Lupu, and J. A. Menendez, "Overexpression of fatty acid synthase gene activates HER1/HER2 tyrosine kinase receptors in human breast epithelial cells," Cell proliferation, vol. 41, no. 1, pp. 59-85, 2008.

[38] F. B. Feng and H. Y. Qiu, "Effects of Artesunate on chondrocyte proliferation, apoptosis and autophagy through the $\mathrm{PI} 3 \mathrm{~K} / \mathrm{AKT} / \mathrm{mTOR}$ signaling pathway in rat models with rheumatoid arthritis," Biomedicine \& pharmacotherapy = Biomedecine \& pharmacotherapie, vol. 102, pp. 1209-1220, 2018. 